\title{
March 2015 Editorial
}

Welcome to the first issue of the Statistical Journal of the IAOS in 2015. In this issue, we'll cover an interview with the Director of the Lesotho Bureau of Statistics, introduce a special section on local area data as well as other interesting topics.

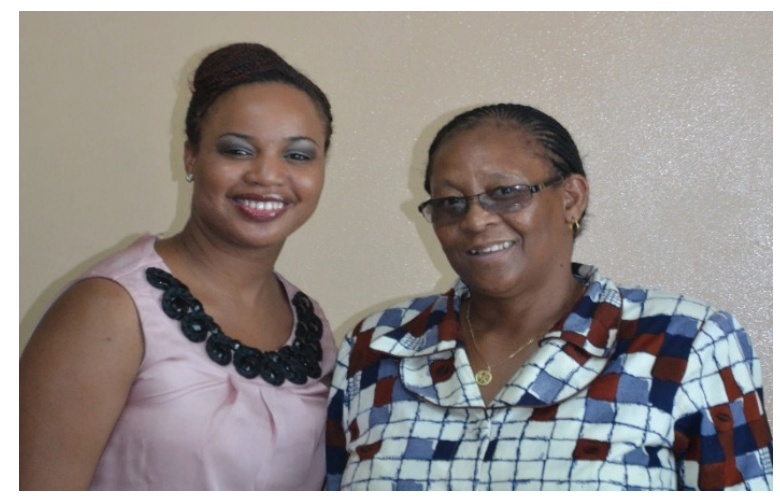

As usual, Kirsten West, as the IAOS Statistical Journal Interview Editor, asked the questions and edited what you have in this Issue. This time she was assisted by Dr. Muriel Dow, pictured on the left above. Dr. Dow is a Resident Technical Advisor at IHM - the Institute of Health Measurement Lesotho. Dr. Dow is also the Africa Editor for our IAOS Statistical Journal.

Pictured above with Dr. Dow, on the right is our interviewee, Liengoane Mothoweso.Lefosa. Ms. Lefosa is the Director of the Bureau of Statistics in the mountain kingdom of Lesotho, Africa. She has just been put up for the prestigious Mahalanobis Award for high statistical achievers in developing countries. During her tenure, she has transformed her Office from a small statistical office to a full-fledged National Statistical Institute (NSI). Part of the reason for this is that during her tenure, Ms. Lefosa has made outstanding contributions towards the national statistical system in her country including the Southern African Development Community (SADC) Strategy for Development of Statistics and the Strategy for Harmonization of Statistics.

\section{Rest of this issue?}

The paper Using G2 to measure income inequality in two Latin American upper middle income countries by Tina Faulkner first appeared in the December 2014 issue of the Journal. Since income inequality issues have been featured in many media settings lately, we sought discussants from Brazil and Mexico for the Faulkner paper. In this issue there is a comment by M.A.P. Bruno from Brazil that appears here, with a rejoinder by Faulkner. There will be a whole separate paper, in a later issue from Mexico.

S. Pierson starts the rest of this Journal with a paper entitled Official Statistics Principles Compared. In March 2014, the UN formally adopted its set of Official Statistical Principles and that inspired the paper shown here. We expect this paper will be the start of a long thread of papers with comments from Official Statisticians around the world. Stay Tuned.

Efficient statistical information system for the state building by Jan Suesser looks at the challenges for official statistics acting in unstable circumstances. Official statisticians are to continuously measure the economical and the social evolutions in their country and in relation with the rest of the world. Especially hard in many parts of the world, including Palestine.

The policeman and the statistician: On the quality of the raw data in official statistics by Anton Färnström reports the results of two studies conducted by the Swedish National Council for Crime Prevention evaluating the quality of the raw data used for the official crime statistics in Sweden. There is also a short comment by S. Granath on the paper.

Next this issue includes three winning papers of Young Statistician Awardees given in DaNang, Viet Nam, last year at the IAOS Statistical Conference. The papers and authors are:

Linking, selecting cut-offs, and examining quality in the Integrated Data Infrastructure (IDI) by Laura $O$ ' Sullivan

Domains similarity models in synthetic estimation by Andrius Čiginas 
Transforming how we produce statistics: An inside perspective by Michelle Feyen

Before turning to the Special Section, we have one last paper, continuing our tradition of publishing papers on the Indigenous. This paper is entitled Back to the basics: Identifying and addressing underlying challenges in achieving high quality and relevant health statistics for indigenous populations in Canada. It is by Janet Smylie and Michelle Firestone. Their paper looks at the double standards with respect to the relevance and quality of statistics for Indigenous populations in Canada.
In their paper there is also a focus on how the current Indigenous data quality challenges including misclassification errors and non-response bias systematically contribute to a significant underestimate of inequities in health determinants, health status, and health care access between Indigenous and non-Indigenous people in Canada.

The March issue of the Journal ends with a long special session from our local area editors Asta Manninen, and Derek Bond. They have written a special introduction and you will find the details on their papers there. Here it is enough to heartily thank them for their highly successful effort. 